\title{
Tessellations Generated by Hyperplanes
}

\author{
Rolf Schneider \\ Mathematisches Institut, Universität Freiburg im Breisgau, Albertstrasse 23b, \\ D-7800 Freiburg im Breisgau, Federal Republic of Germany
}

\begin{abstract}
Let $\mathscr{H}$ be a locally finite system of hyperplanes in $\mathbb{R}^{d}$ with the property that the cells of the induced cell complex decomposition $\mathscr{T}$ of $\mathbb{R}^{d}$ have uniformly bounded diameters. If $\mathscr{H}$ is simple and the density of the vertices in $\mathscr{T}$ exists, then the density of the $k$-cells in $\mathscr{T}$ exists and can be given explicitly $(k=1, \ldots, d)$. Also, the mean number of $j$-faces of the $k$-cells in $\mathscr{T}$ exists and can be calculated. For certain nonsimple systems $\mathscr{H}$, corresponding inequalities are obtained.
\end{abstract}

\section{Introduction}

We consider a system $\mathscr{H}$ of hyperplanes in $d$-dimensional Euclidean space $\mathbb{R}^{d}$ and assume that $\mathscr{H}$ is locally finite, that is, every bounded subset of $\mathbb{R}^{d}$ meets only finitely many elements of $\mathscr{K}$. Such a system generates, in the obvious way by intersections, sets $\mathscr{L}_{k}$ of $k$-dimensional flats and sets $\mathscr{C}_{k}$ of $k$-dimensional cells $(k=0,1, \ldots, d)$. By the tessellation $\mathscr{T}$ generated by $\mathscr{H}$ we understand here the induced cell complex. As a second assumption on $\mathscr{H}$ we impose the condition that the diameters of the cells of $\mathscr{T}$ are bounded by some constant $\delta$.

Let $B=\left\{x \in \mathbb{R}^{d}:\|x\| \leq 1\right\}$ be the unit ball of $\mathbb{R}^{d}$, and for $r>0$ define

$$
\mathscr{C}_{k}(r)=\left\{Z \in \mathscr{C}_{k}: Z \subset r B\right\}
$$

If $\varphi$ is a real function defined on $\mathscr{C}_{k}$, we write

$$
\varphi_{k}(r)=\sum_{Z \in \mathscr{C}_{k}(r)} \varphi(Z)
$$

and we define the $k$-cell density of $\varphi$ by

$$
\varphi_{k}=\lim _{r \rightarrow \infty} \varphi_{k}(r) / V(r B)
$$


if the limit exists; here $V$ denotes the volume. In particular, from the function $g \equiv 1$ we get $g_{k}$, the density of $k$-cells in $\mathscr{T}$.

For a convex polytope $P, f_{j}(P)$ denotes the number of $j$-faces. We write $\left(f_{j}\right)_{k}=f_{j k}$ and define

$$
N_{k j}=\lim _{r \rightarrow \infty} f_{j k}(r) / g_{k}(r)
$$

for $k=1, \ldots, d$ and $j=0, \ldots, k$, if the limit exists. Thus $N_{k j}$ can be interpreted as the mean number of $j$-faces of the $k$-cells in $\mathscr{T}$.

Of course, our two assumptions on $\mathscr{H}$, local finiteness and uniform boundedness of the cell diameters, do not imply that any of the densities $g_{j}$ or mean values $N_{k j}$ exist. However, if we assume that $\mathscr{H}$ is simple, then the assumption that the vertex density $g_{0}$ exists, already has strong implications. $\mathscr{H}$ is called simple if no hyperplane of $\mathscr{H}$ contains a nonempty fiat determined by other hyperplanes of $\mathscr{H}$.

Theorem 1. Suppose that $\mathscr{H}$ is simple. If the vertex density $g_{0}$ exists, then all densities $g_{k}$ and mean values $N_{k j}$ exist, and they satisfy

$$
\begin{gathered}
g_{k}=\left(\begin{array}{l}
d \\
k
\end{array}\right) g_{0}, \\
N_{k j}=2^{k-j}\left(\begin{array}{l}
k \\
j
\end{array}\right)
\end{gathered}
$$

for $k=1, \ldots, d$ and $j=0, \ldots, k$

The numbers occurring in (1) and (2) are, of course, those of the cubical tessellation, generated by hyperplanes parallel to the coordinate hyperplanes.

The present considerations were motivated by a paper of Mecke [3], who proved results analogous to (1) and (2) for random tessellations generated by stationary random hyperplane systems. In his case, the numbers $g_{k}$ are intensities (describing intensity measures in a stationary situation) and $N_{k j}$ is the mean number of $j$-faces of the "typical" $k$-cell (in a probabilistic sense which can be made precise by using Palm distributions). The fact that his results do not require special assumptions on the underlying probability distribution seems to indicate that they have a purely geometric kernel rather than being of a truly stochastic nature. Our Theorem 1 can be considered as expressing such a geometric kernel. It is not implied by Mecke's theorem, and a deduction of Mecke's theorem from ours would, if possible, probably require rather deep results, in contrast to Mecke's simple proof. The central idea of the proof below, the use of Gram's theorem, is taken from Mecke's paper. An essential feature of our result is the fact that the existence of $g_{0}$ implies the existence of the other densities and mean values, while in Mecke's case no existence problems occurred because of the stationarity assumptions.

Mecke considered only simple hyperplane systems, but here we will also study the general case and end up with some inequalities. 


\section{Proof of the Result}

We start with a system $\mathscr{H}$ which is not necessarily simple. Let $k \in\{1, \ldots, d\}$. For a cell $Z$ of $\mathscr{T}$, we denote by $e_{k}(Z)$ and $c_{k}(Z)$ the number of $k$-flats in $\mathscr{L}_{k}$, respectively of $k$-cells in $\mathscr{C}_{k}$, which contain $Z$. For $j \in\{0, \ldots, k\}$ and a polytope $P$, let

$$
S_{j}(P)=\sum_{F \in \mathcal{F}(P)} \beta(P, F)
$$

where $\beta(P, F)$ is the (normalized) internal angle of $P$ at its face $F$. We have

$$
\begin{aligned}
& \sum_{P \in \mathscr{G}_{k}(r)} S_{j}(P)=\sum_{P \in \mathscr{G}_{k}(r)} \sum_{\substack{F \in \mathscr{C}_{j}(r) \\
F \in P}} \beta(P, F) \\
& =\sum_{F \in \mathscr{C}_{j}(r)} \sum_{F \in P \in \mathscr{C}_{k}(r)} \beta(P, F) \\
& =\sum_{F \in \mathscr{C}_{j}(r)}\left[\sum_{F \subset P \in \mathscr{C}_{k}} \beta(P, F)-\sum_{\substack{F \subset P \in \mathscr{C}_{k} \\
P \neq>B}} \beta(P, F)\right] \\
& =e_{k j}(r)-\sum_{F \in \mathscr{C}_{j}(r)} \sum_{\substack{F \subset P_{\mathbb{E}} \mathscr{G}_{k} \\
P \not \subset B}} \beta(P, F),
\end{aligned}
$$

since

$$
\sum_{\substack{P \in \mathscr{C}_{k} \\ F \subset P \subset L}} \beta(P, F)=1
$$

if $F \subset L \in \mathscr{L}_{k}$, and there are $e_{k}(F)$ such flats $L$. Now Gram's relation (see, e.g., Grünbaum [1])

$$
\sum_{j=0}^{k}(-1)^{j} S_{j}(P)=0
$$

gives

$$
\sum_{j=0}^{k}(-1)^{j} e_{k j}(r)=a_{k}(r)
$$

with

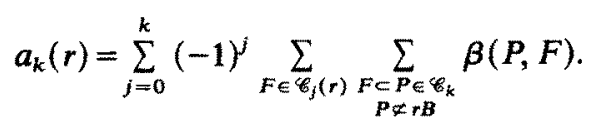

Since $e_{d}=1$, we have $e_{d j}(r)=g_{j}(r)$, and from $e_{k}(F)=1$ if $k=\operatorname{dim} F$ it follows that $e_{k k}(r)=g_{k}(r)$. Thus $(3)$ reduces to

$$
\begin{gathered}
{\left[\sum_{j=0}^{k-1}(-1)^{j} e_{k j}(r)\right]+(-1)^{k} g_{k}(r)=a_{k}(r), \quad k=1, \ldots, d-1,} \\
\sum_{j=0}^{d}(-1)^{j} g_{j}(r)=a_{d}(r) .
\end{gathered}
$$


The numbers $a_{k}(r)$ can be estimated as follows. If $F \in \mathscr{C}_{j}(r)$ is such that there exists $P \in \mathscr{C}_{k}$ with $F \subset P$ and $P \not \subset r B$, then $F \cap(r-\delta) B=\varnothing$, since the diameter of $P$ is at most $\delta$. It follows that

$$
\begin{aligned}
\left|a_{k}(r)\right| & \leq \sum_{j=0}^{k-1} \sum_{F \in \mathscr{C}_{j}(r) \backslash \mathscr{C}_{j}(r-\delta)} e_{k}(F) \\
& =\sum_{j=0}^{k-1}\left[e_{k j}(r)-e_{k j}(r-\delta)\right],
\end{aligned}
$$

in particular,

$$
\left|a_{d}(r)\right| \leq \sum_{j=0}^{d-1}\left[g_{j}(r)-g_{j}(r-\delta)\right]
$$

If we now assume that the density

$$
e_{k j}=\lim _{r \rightarrow \infty} e_{k j}(r) / V(r B)
$$

exists, then

$$
\lim _{r \rightarrow \infty}\left[e_{k j}(r)-e_{k j}(r-\delta)\right] / V(r B)=0
$$

Hence, from (5) we deduce:

Proposition 1. If all densities $e_{k j}(k=1, \ldots, d-1 ; j=0, \ldots, k-1)$ exist, then the densities $g_{k}(k=1, \ldots, d-1)$ also exist and

$$
\left[\sum_{j=0}^{k-1}(-1)^{j} e_{k j}\right]+(-1)^{k} g_{k}=0
$$

If, moreover, the density $g_{0}$ exists, then $g_{d}$ also exists, and

$$
\sum_{j=0}^{d}(-1)^{j} g_{j}=0
$$

Unfortunately, the necessary existence assumptions are so strong that this result seems of little interest. In the simple case, however, the situation is different. If $\mathscr{H}$ is simple, then

$$
e_{k}(F)=\left(\begin{array}{l}
d-j \\
d-k
\end{array}\right) \quad \text { if } \quad \operatorname{dim} F=j
$$

hence

$$
e_{k j}(r)=\left(\begin{array}{c}
d-j \\
d-k
\end{array}\right) g_{j}(r)
$$

and system (5) now reads

$$
\sum_{j=0}^{k}(-1)^{j}\left(\begin{array}{l}
d-j \\
d-k
\end{array}\right) g_{j}(r)=a_{k}(r), \quad k=1, \ldots, d .
$$


Its solution is given by

$$
g_{k}(r)=\left(\begin{array}{l}
d \\
k
\end{array}\right) g_{0}(r)+\sum_{i=1}^{k}(-1)^{i}\left(\begin{array}{c}
d-i \\
d-k
\end{array}\right) a_{i}(r)
$$

In this case, we estimate $a_{k}(r)$ differently. Each cell $F \in \mathscr{C}_{j}(r) \backslash \mathscr{C}_{j}(r-\delta)$ contains a vertex in $r B \backslash(r-\delta) B$. Each such vertex is contained in $2^{j}\left(d_{d-j}^{d}\right) j$-cells, and each $j$-cell in $2^{k-j}\left(\begin{array}{c}d-j \\ d-k\end{array}\right) k$-cells, hence

$$
\left|a_{k}(r)\right| \leq 2^{k} \sum_{j=0}^{k-1}\left(\begin{array}{c}
d \\
d-j
\end{array}\right)\left(\begin{array}{c}
d-j \\
d-k
\end{array}\right)\left[g_{0}(r)-g_{0}(r-\delta)\right] .
$$

It is now clear that the existence of $g_{0}$ alone implies the existence of $g_{k}$ and equality (1).

Now let $\mathscr{H}$ again be not necessarily simple, and let $k \in\{0, \ldots, d\}$ and $j \in$ $\{0, \ldots, k\}$ be given. We have

$$
\begin{aligned}
f_{j k}(r) & =\sum_{P \in \mathscr{C}_{k}(r)} f_{j}(P)=\sum_{P \in \mathscr{C}_{k}(r)} \sum_{\substack{F \in \mathscr{C}_{j} \\
F \subset P}} 1 \\
& =\sum_{F \in \mathscr{C}_{j}} \sum_{F \subset P \in \mathscr{C}_{k}(r)} 1=\sum_{F \in \mathscr{C}_{j}(r)}\left[\sum_{F \in P \in \mathscr{C}_{k}} 1-\sum_{\substack{F \in P \in \mathscr{C}_{k} \\
P \mathscr{P} \not r B}} 1\right] \\
& =c_{k j}(r)-b_{k j}(r)
\end{aligned}
$$

with

$$
\begin{aligned}
b_{k j}(r) & =\sum_{F \in \mathscr{C}_{j}(r)} \sum_{\substack{F \in P \in \mathscr{C}_{k} \\
\boldsymbol{P} \notin \boldsymbol{B} \boldsymbol{B}}} 1 \leq \sum_{F \in \mathscr{C}_{j}(r) \backslash \mathscr{C}_{j}(r-\delta)} c_{k}(F) \\
& =c_{k j}(r)-c_{k j}(r-\delta) .
\end{aligned}
$$

Since the cell diameters in $\mathscr{T}$ are uniformly bounded, it is easy to see that $g_{k} \neq 0$ (if $g_{k}$ exists) for $k=0, \ldots, d$. Writing the above in the form

$$
\frac{g_{k}(r)}{V(r B)} \frac{f_{j k}(r)}{g_{k}(r)}=\frac{c_{k j}(r)}{V(r B)}-\frac{b_{k j}(r)}{V(r B)}
$$

we deduce:

Proposition 2. Let $k \in\{1, \ldots, d\}$ and $j \in\{0, \ldots, k\}$ be given. If the densities $c_{k j}$ and $g_{k}$ exist, then the mean value $N_{k j}$ exists and satisfies

$$
g_{k} N_{k j}=c_{k j}
$$


If, now, $\mathscr{H}$ is simple, we have

$$
c_{k}(F)=2^{k-j}\left(\begin{array}{c}
d-j \\
d-k
\end{array}\right) \quad \text { if } \quad \operatorname{dim} F=j
$$

hence

$$
c_{k j}(r)=2^{k-j}\left(\begin{array}{l}
d-j \\
d-k
\end{array}\right) g_{j}(r)
$$

Thus the existence of $g_{0}$ implies that of each $c_{k j}$, and from (1) and (10) we obtain (2). Theorem 1 is proved.

\section{Line-Simple Systems}

Between the case of totally general systems $\mathscr{H}$, which requires too many assumptions to be of interest, and the simple case, there is another specialization which deserves some further study, namely, systems $\mathscr{H}$ where the deviation from simplicity occurs only at vertices. We say that $\mathscr{H}$ is line-simple if no hyperplane of $\mathscr{H}$ contains a flat of positive dimension determined by other hyperplanes of $\mathscr{H}$. In this case we have

$$
\begin{aligned}
& e_{k j}(r)=\left(\begin{array}{c}
d-j \\
d-k
\end{array}\right) g_{j}(r) \quad \text { for } \quad j>0, \\
& c_{k j}(r)=2^{k-j}\left(\begin{array}{l}
d-j \\
d-k
\end{array}\right) g_{j}(r) \quad \text { for } \quad j>0 .
\end{aligned}
$$

For $F \in C_{0}$ we define $\eta_{k}(F), \zeta_{k}(F)$ by means of

$$
\begin{aligned}
& e_{k}(F)=\left(\begin{array}{l}
d \\
k
\end{array}\right)\left[1+\eta_{k}(F)\right], \\
& c_{k}(F)=2^{k}\left(\begin{array}{l}
d \\
k
\end{array}\right)\left[1+\zeta_{k}(F)\right] .
\end{aligned}
$$

We may call $\eta_{k}(F), \zeta_{k}(F)$ the (normalized) $k$-flat excess, respectively $k$-cell excess, at the vertex $F$. Since

$$
\begin{aligned}
& e_{k 0}(r)=\left(\begin{array}{l}
d \\
k
\end{array}\right)\left[g_{0}(r)+\eta_{k 0}(r)\right], \\
& c_{k 0}(r)=2^{k}\left(\begin{array}{l}
d \\
k
\end{array}\right)\left[g_{0}(r)+\zeta_{k 0}(r)\right],
\end{aligned}
$$

system (5) now reads

$$
\left(\begin{array}{l}
d \\
k
\end{array}\right) \eta_{k 0}(r)+\sum_{j=0}^{k}(-1)^{j}\left(\begin{array}{l}
d-j \\
d-k
\end{array}\right) g_{j}(r)=a_{k}(r), \quad k=1, \ldots, d,
$$


with $\eta_{d 0}(r)=0$. This yields

$$
g_{k}(r)=\left(\begin{array}{l}
d \\
k
\end{array}\right) g_{0}(r)+\sum_{i=1}^{k}(-1)^{i}\left(\begin{array}{c}
d-i \\
d-k
\end{array}\right)\left[a_{i}(r)-\left(\begin{array}{l}
d \\
i
\end{array}\right) \eta_{i 0}(r)\right]
$$

Using estimate (6) together with (11) and (13) we see (by induction, in this case) that the existence of the densities $g_{0}, \eta_{10}, \ldots, \eta_{(d-1) 0}$ implies the existence of $g_{1}, \ldots, g_{d}$ and the equations

$$
g_{k}=\left(\begin{array}{l}
d \\
k
\end{array}\right) g_{0}-\sum_{i=1}^{k}(-1)^{i}\left(\begin{array}{c}
d-i \\
d-k
\end{array}\right)\left(\begin{array}{l}
d \\
i
\end{array}\right) \eta_{i 0}
$$

Let $k \in\{1, \ldots, d\}$. If the densities $g_{0}, g_{k}$, and $\zeta_{k 0}$ exist, then (by (10) and (14)) the mean value $N_{k 0}$ exists and

$$
g_{k} N_{k 0}=2^{k}\left(\begin{array}{l}
d \\
k
\end{array}\right)\left[g_{0}+\zeta_{k 0}\right]
$$

If $j \in\{1, \ldots, k\}$ and the densities $g_{j}$ and $g_{k}$ exist, then (by (10) and (12)) the mean value $N_{k j}$ exists and

$$
g_{k} N_{k j}=2^{k-j}\left(\begin{array}{c}
d-j \\
d-k
\end{array}\right) g_{j}
$$

In the special case $d=2$, we have $c_{2}(F)=2 e_{1}(F)$ for $F \in C_{0}$, hence $\zeta_{20}(r)=$ $\eta_{10}(r)$. Therefore, the existence of the densities $g_{0}$ and $\eta_{10}$ implies

$$
\begin{gathered}
g_{1}=2 g_{0}+2 \eta_{10}, \\
g_{2}=g_{0}+2 \eta_{10} \\
N_{20}=4\left[1-\frac{\nu_{10}}{g_{0}+2 \eta_{10}}\right] \leq 4
\end{gathered}
$$

Thus in the plane the mean vertex number of the 2 -cells of $\mathscr{T}$ is at most 4, with equality if and only if the density of the nonsimple vertices, each one counted with its excess (number of lines through it minus two) as multiplicity, is zero.

We want to show that simple systems in higher dimensions have a similar extremal property among the line-simple systems. For that purpose we first prove:

Proposition 3. Let $\mathscr{H}$ be line-simple and $k \in\{1, \ldots, d\}$. Then for any vertex $F \in \mathscr{C}_{0}$

$$
\sum_{i=1}^{k}(-1)^{i}\left(\begin{array}{c}
d-i \\
d-k
\end{array}\right) e_{i}(F)+2^{-k} c_{k}(F) \leq 0
$$


and, for $j \in\{1, \ldots, k\}$,

$$
\left(\begin{array}{l}
d \\
j
\end{array}\right) \sum_{i=1}^{k}(-1)^{i}\left(\begin{array}{l}
d-i \\
d-k
\end{array}\right) e_{i}(F)-\left(\begin{array}{l}
d \\
k
\end{array}\right) \sum_{i=1}^{j}(-1)^{i}\left(\begin{array}{l}
d-i \\
d-j
\end{array}\right) e_{i}(F) \leq 0 .
$$

For the proof let $N$ denote the number of hyperplanes in a system $\mathscr{H}$ that pass through $F$. Since $\mathscr{H}$ is line-simple, these hyperplanes are in general position. Therefore,

$$
e_{i}(F)=\left(\begin{array}{c}
N \\
d-i
\end{array}\right)
$$

and, by a well-known formula going back, in special cases, to Steiner and Schläfli (see Grünbaum [2] and Zaslavsky [4] for references),

$$
c_{k}(F)=2\left(\begin{array}{c}
N \\
d-k
\end{array}\right) \sum_{i=0}^{k-1}\left(\begin{array}{c}
N-d+k-1 \\
i
\end{array}\right)
$$

Writing

$$
\begin{aligned}
\sum_{i=1}^{k}(-1)^{i}\left(\begin{array}{c}
d-i \\
d-k
\end{array}\right)\left(\begin{array}{c}
N \\
d-i
\end{array}\right) & =\varphi(N, d, k), \\
\left(\begin{array}{c}
N \\
d-k
\end{array}\right) \sum_{i=0}^{k-1}\left(\begin{array}{c}
N-d+k-1 \\
i
\end{array}\right) & =\psi(N, d, k)
\end{aligned}
$$

for $k \geq 1$ and $N \geq d \geq 1$, we have to prove that

$$
2^{k-1} \varphi(N, d, k)+\psi(N, d, k) \leq 0
$$

for $k \leq d$ and

$$
\left(\begin{array}{l}
d \\
j
\end{array}\right) \varphi(N, d, k)-\left(\begin{array}{l}
d \\
k
\end{array}\right) \varphi(N, d, j) \leq 0
$$

for $j<k$. One gets

$$
\varphi(N+1, d+1, k)=\varphi(N, d, k)-\left(\begin{array}{l}
N \\
d
\end{array}\right)\left(\begin{array}{c}
d \\
d+1-k
\end{array}\right)
$$

which can be used to prove the identity

$$
\varphi(N, d, k)=-\frac{k}{N-d+k}\left(\begin{array}{l}
N \\
d
\end{array}\right)\left(\begin{array}{l}
d \\
k
\end{array}\right)
$$

by induction; the case $d=1$ is trivial. Therefore, the left-hand side of (22) is equal to

$$
\left(\begin{array}{l}
N \\
d
\end{array}\right)\left(\begin{array}{l}
d \\
j
\end{array}\right)\left(\begin{array}{l}
d \\
k
\end{array}\right) \frac{(k-j)(d-N)}{(N-d+j)(N-d+k)} \leq 0
$$

which proves (22). 
For the proof of $(21)$ we first assume that $k=d$. In that case we have to show that

$$
\sum_{i=0}^{d-1}\left(\begin{array}{c}
N-1 \\
i
\end{array}\right) \leq 2^{d-1}\left(\begin{array}{c}
N-1 \\
d-1
\end{array}\right)
$$

for $N \geq d$. Denoting the left-hand side by $\alpha(N, d)$, we get

$$
\alpha(N+1, d+1)=2 \alpha(N, d)+\left(\begin{array}{c}
N-1 \\
d
\end{array}\right)
$$

which yields (24) by induction, the case $d=1$ being trivial. Also, the general inequality (21) is trivial for $d=1$. Suppose (21) has been proved in dimension $d$. For $k \leq d$ we get

$$
\psi(N+1, d+1, k)=\frac{N+1}{d-k+1} \psi(N, d, k)
$$

Together with (23) and the induction hypothesis, this yields

$$
2^{k-1} \varphi(N+1, d+1, k)+\psi(N+1, d+1, k) \leq 0 .
$$

For $k=d+1$, this inequality has already been proved above. This completes the inductive proof of (21) and thus the proof of Proposition 3.

Now, using the definition of $\eta_{i}(F)$ and $\zeta_{k}(F)$ together with an identity for binomial coefficients, we can write (19) in the form

$$
H_{k}(F)+\left(\begin{array}{l}
d \\
k
\end{array}\right) \zeta_{k}(F) \leq 0
$$

with

$$
H_{k}(F)=\sum_{i=1}^{k}(-1)^{i}\left(\begin{array}{c}
d-i \\
d-k
\end{array}\right)\left(\begin{array}{l}
d \\
i
\end{array}\right) \eta_{i}(F)
$$

Summing over the vertices in $r B$, we get

$$
H_{k 0}(r)+\left(\begin{array}{l}
d \\
k
\end{array}\right) \zeta_{k 0}(r) \leq 0
$$

and hence, if the densities involved exist,

$$
\left(\begin{array}{l}
d \\
k
\end{array}\right)\left(g_{0}+\zeta_{k 0}\right) \leq g_{k}
$$

by (16), which by (17) implies $N_{k 0} \leq 2^{k}$. 
Similarly, from (20) we get

$$
\left(\begin{array}{l}
d \\
j
\end{array}\right) H_{k 0}(r)-\left(\begin{array}{l}
d \\
k
\end{array}\right) H_{j 0}(r) \leq 0
$$

for $j \leq k$ and hence

$$
\left(\begin{array}{l}
d-j \\
d-k
\end{array}\right) g_{j} \leq\left(\begin{array}{l}
k \\
j
\end{array}\right) g_{k}
$$

from (16). By (18) this implies $N_{k j} \leq 2^{k-j}\left({ }_{j}^{k}\right)$.

We collect our results in the following theorem:

Theorem 2. Suppose that $\mathscr{H}$ is line-simple and that the vertex density $g_{0}$ and the flat excess densities $\eta_{10}, \ldots, \eta_{(d-1) 0}$ exist. Then the densities $g_{1}, \ldots, g_{d}$ exist and are given by (16). For $j \in\{1, \ldots, k\}$ one has

$$
N_{k j}=2^{k-j}\left(\begin{array}{l}
d-j \\
d-k
\end{array}\right) g_{j} / g_{k} \leq 2^{k-j}\left(\begin{array}{l}
k \\
j
\end{array}\right) .
$$

If, moreover, the $k$-cell excess density $\zeta_{k 0}$ exists, then

$$
N_{k 0}=2^{k}\left(\begin{array}{l}
d \\
k
\end{array}\right)\left(g_{0}+\zeta_{k 0}\right) / g_{k} \leq 2^{k}
$$

\section{References}

1. B. Grünbaum, Convex Polytopes, Interscience, London, 1967.

2. B. Grünbaum, Arrangements of hyperplanes, Proceedings of the Second Louisiana Conference on Combinatorics, Graph Theory, and Computing, Baton Rouge, 1971.

3. J. Mecke, Random tessellations generated by hyperplanes, in Stochastic Geometry, Geometric Statistics, Stereology (R. Ambartzumian and W. Weil, eds.), 104-109, Teubner, Leipzig, 1984.

4. T. Zaslavsky, Facing up to arrangements: face count formulas for partitions of space by hyperplanes, Mem. Amer. Math. Soc. 1 (1975), 1-102.

Received September 22, 1986. 